\title{
IMPLICACIONES PSICOPEDAGÓGICAS DE UN DESARROLLO MORAL INTEGRO: LA EDUCACIÓN HOLÍSTICA
}

Alejandra Cortés Pascual

Universidad de Zaragoza, España

Para mis padres, Angelines y Agustín.

"No hay nada más absurdo para Aristóteles que saber en qué consiste el bien pero no saber cómo ponerlo en práctica: practicando la justicia nos hacemos justos"

(Muñoz Redón, Filosofía de la felicidad, 1999)

\section{INTRODUCCIÓN}

El desarrollo moral como integral constituye el análisis teórico de este estudio. Este cometido supone una fundamentación de la moral que nos conduce a la autorrealización en plenitud y su concomitante vivencia como felicidad y, ésta como criterio de lo moral porque tiende al desarrollo íntegro y auténtico del ser humano en su integridad o totalidad.

Para ello, este trabajo indaga sobre dos cuestiones, entre las que no tiene por qué existir una frontera claramente definida y sí una relación estrecha entre ellas, como son; ¿qué componentes existen en el desarrollo moral integro? y ¿qué tipo de educación responde a este cometido? El ápice o punto culminante, a la vez que dificultoso por la complejidad del tema, podría ser dar una respuesta clara y rotunda de estos dos interrogantes. En dicha empresa se trabaja, y así este estudio se plantea para aportar datos teóricos y avanzar en las indagaciones.

\section{EL RECORRIDO TEÓRICO DE LO MORAL Y DE LA INTEGRIDAD DEL SUJETO}

Este camino describe vericuetos tales que aúnan aspectos diferentes para la comprensión de la totalidad de la persona, como el razonamiento, el carácter, el compromiso, la sensibilidad o inteligencia emocional, la compasión y la sabiduría vital.

Frecuentemente las teorías psicopedagógicas que se ocupan del desarrollo moral son teorías sectoriales sobre algunos aspectos, elementos o particularidades comprendidas realmente en el desarrollo moral. No obstante, se hace ahora evidente la necesidad de contar con una teoría integral del desarrollo moral que, efectivamente, integrase los distintos modelos, planteamientos y aspectos de las teorías parciales, pero teniendo en cuenta, claro está, que esta teoría no podría reducirse a un mero eclecticismo, sino que habría de consistir en una nueva praxis distinta, con su propia fundamentación, y pensada y desarrollada de acuerdo con las exigencias epistemológicas particulares de su nuevo contenido. 
Una teoría psicopedagógica del desarrollo moral íntegro sólo tiene validez teórica si no se plantea cómo recopilar y compatibilizar distintos planteamientos particulares, distintas metodologías y teorías sectoriales previas en una teoría total que difícilmente podría tener al menos unidad sistemática y consistencia lógica. Para ello es necesario contar previamente con un planteamiento del desarrollo moral íntegro de las personas, de los individuos, entendido como desarrollo humano y moral.

Esta teoría psicopedagógica integral del desarrollo moral, por lo tanto, sólo podrá tener un desarrollo epistemológico válido si se transforma y se desarrolla como teoría del desarrollo íntegro del ser humano porque éste es, precisamente, su reciente objeto de estudio.

La psicología y la pedagogía han de ocuparse del análisis de las virtudes, actitudes y normas teniendo en cuenta su función y condicionamiento en el desarrollo moral como desarrollo humano íntegro, y han de ofrecer propuestas educativas considerando la naturaleza racional y emocional del hombre, y la dimensión cultural, social y comunitaria del ser humano, es decir, de su plena auto-co-realización y su felicidad. Como apunta Gultman (2001), todas las personas tienen el derecho de ser felices y la educación debe de dar alternativas a este proceso y objetivo vital.

Dicho de otra manera, existe cada vez más la necesidad de una perspectiva de estudio del desarrollo moral global e integradora, y que atienda a aspectos del campo cognitivo, afectivo y contextual. Y esta visión es la que imprescindiblemente se debe adoptar, porque desde estos tres ámbitos, se explicará de manera completa el desarrollo y comportamiento o conducta moral de los individuos. Con este mismo discurso, Lickona (1992) estudia que para establecer un puente de unión entre lo que se piensa que es correcto y una actuación acorde con ese razonamiento, se debe prestar atención al pensamiento, a la afectividad y a la acción.

\section{LOS PRECEDENTES DEL DESARROLLO MORAL HASTA LA EDUCACIÓN} INTEGRAL

Aunque el desarrollo moral se ha tratado de abordar desde diferentes modelos o enfoques psicológicos y educativos, es el enfoque cognitivo-evolutivo de Kohlberg (1992) al que más atención se le ha prestado. Al aludir al término cognitivo, se entiende que el desarrollo moral tiene sus bases en la estimulación del razonamiento de la persona acerca de cuestiones y decisiones respecto a situaciones de relación interpersonal. El papel del pensamiento es importante, y sobre todo el cómo y el porqué el sujeto justifica y razona sus decisiones morales. $\mathrm{Y}$ es un modelo evolutivo porque propone una secuencia de desarrollo moral a través de tres niveles; preconvencional, convencional y postconvencional, que se suceden progresivamente desde un razonamiento menos equilibrado y maduro hasta uno más avanzado, y a su vez cada nivel se subdivide en estadios o etapas morales.

A pesar de que el enfoque kohlberiano es el marco teórico del desarrollo moral por antonomasia, presenta algunas limitaciones (Aierbe, Cortés y Medrano, 2001). La idea que resume todas estas críticas es la de que el marco teórico de Kohlberg se centra básicamente y excesivamente en la estructura del pensamiento. Aunque el enfoque de Kohlberg necesita modificaciones y ampliaciones, éste es siempre 
necesario para tratar el crecimiento moral y, en general, comprender el desarrollo y madurez personal, puesto que el razonamiento es una parte esencial en la atención de lo moral. Para que un individuo sea capaz de escoger mejor una opción entre las posibles, debe conocer los principios morales que rigen el juicio moral adecuado en torno al seno de la comunidad social en la que vive.

Si quizás la teoría kohlberiana presupone un cierto reduccionismo sobre lo moral como globalidad, una conceptualización más amplia se encuentra cesde la construcción terminológica de "carácter moral" que hace hincapié en la propia persona y en la que se valora la comunidad social que posibilita una formación de este carácter. La preocupación por este factor conduce a un marco comprehensivo donde el juicio y la práctica moral definen a la personalidad moral. Este modelo tiene sus bases en el contexto norteamericano promovido por una asociación cuyo fin es la educación del individuo como agente moral y en donde es importante la influencia de instituciones escolares y otras como las familiares. Lickona (1991) lo define como disposiciones estables para responder a situaciones de modo moral, manifestadas en rasgos palpables de amabilidad, honestidad, responsabilidad y respeto generalizado por los demás. Por esta razón, define el carácter de los ciudadanos como la medida en que una masa crítica de individuos poseen y encuentran su identidad en la sociedad y actúan con base en una visión moral compartida.

En muchos casos se basó en un modelo de virtud derivado del trabajo de Aristóteles. El argumento básico consistió en que la conducta moral se debe aprender, practicar y en última instancia interiorizar, como un carácter o virtud. Es necesario demostrar y recompensar la conducta adecuada, de modo que se convierta en un hábito. Es el acto habitual el que deriva en el rasgo de carácter. Ello crea un círculo interesante con la conducta; la conducta correcta se modela y conforma (por los métodos tradicionales de aprendizaje), lo cual conduce a tendencias interiorizadas que producen comportamientos correctos. De ahí que podamos apreciar claramente la relación entre los dos primeros elementos de la anatomía moral: la conducta y el carácter.

La educación del carácter es ampliamente compatible con las cuestiones de educación en valores. Por tanto, el proceso de enseñanza-aprendizaje del carácter necesita ir más allá de una simple defensa de tendencias de conducta deseada. La educación del carácter puede y debería incluir un planteamiento sobre la interiorización u ósmosis de creencias, pero hay que ir más lejos fomentando hábitos morales, tendencias de conducta, que acaben siendo de por sí rasgos de carácter o virtudes. Lickona (1991b) describe una práctica escolar en el contexto norteamericano por la que un profesor de quinto grado programa regularmente un tipo de apreciación, tiempo en el que los estudiantes reconocen públicamente cómo les han ayudado a los demás. En otro trabajo de Lickona (1996) sugiere unos principios para enseñar a los alumnos lo qué es correcto (cognición), el cómo comprometerse con lo bueno (afectividad) y la forma de actuar de acuerdo a esa virtud de la bondad (comportamiento). Incide en que la escuela debe estar comprometida con el desarrollo de la educación del carácter con un curriculum que respete y tolere la diversidad de los alumnos al promover valores éticos como son la responsabilidad, la honestidad, el respeto hacía sí mismo y hacía los demás.

Ello genera un hábito que se encamina hacia el respeto por el otro. Un ejemplo interesante de cómo la educación del carácter es compatible y se confunde a la vez con la educación en valores lo constituye el 
uso de la literatura para promover el desarrollo del carácter. Bennett (1991) ha creado una antología muy popular de lecturas denominada «El libro de las virtudes» (1993), que pretende ayudar a los padres a promover el carácter. Parece probable pensar, desde nuestra anatomía moral, que la lectura puede contribuir a ejercer un importante impacto en el desarrollo de la personalidad (es decir, del carácter). Por otra parte, puede presentar ciertamente un impacto en el modelado de actitudes y creencias (es decir, de valores). Ello significa que la enseñanza de valores debería ser una parte de la educación del carácter, ya que la reflexión sobre los valores propios es un aspecto crítico de la transformación de hábitos en carácter, junto a otros procedimientos, que se verán conforme se avanza en la lectura del presente texto, que son necesarios para el desarrollo de la empatía, la compasión, la resolución de dilemas morales, la sensibilidad y el compromiso con lo social.

Para Walker (1999), la educación del carácter está adquiriendo una perspectiva integradora, promoviendo los valores éticos fundamentales para la formación de la buena persona.

Una consecuencia directa de esta visión de la formación del carácter, es el estudio de la propia realidad biográfica a través de la narración de los propios individuos. La construcción de la personalidad moral es parte de un proceso de adaptación a la sociedad y a uno mismo en el que se forma la conciencia moral autónoma y en el que cada persona elabora su biografía o trayectoria vital. En esta línea de narrativa, Colby y Damon (1994) pretenden recoger, mediante entrevistas, las historias de vida de personas moralmente ejemplares. Estas entrevistas de Colby y Damon intentan unificar factores cognoscitivos y emocionales y la manera en cómo actúan al respecto, además de tener en cuenta la situación y el contexto donde se ubica la persona (Papalia y Wendkos, 1997). En la investigación, llevada a cabo por estas autoras, estudiaron 23 sujetos de "ejemplo de moral", personas que mostraron un grado elevado de moral en su vida diaria. Los criterios para identificar a estas personas se obtuvieron mediante la colaboración de "expertos identificadores" (filósofos, historiadores, pensadores religiosos) y fueron los siguientes: a) actuar de manera consistente según los propios ideales; b) buena voluntad para arriesgar el propio interés; c) inspirar en otros la acción moral; y d) un sentido de humildad y falta de interés en alimentar el propio ego.

Aierbe, Cortés y Medrano (2001) adaptan algunas de las cuestiones utilizadas en la entrevista de Colby y Damon, presentándolas previamente y como complementarias a la indagación de los dilemas reales de los sujetos. Estas preguntas están referidas a tres ámbitos vitales: las preocupaciones o intereses, los éxitos y los fracasos. Además se añade una cuestión a los individuos entrevistados sobre la idea o concepción que tenían de qué es ser buena persona.

Este trabajo constituye una forma de explorar en los sucesos idiosincrásicos desde la perspectiva narrativa, que por otra parte, debe irse desarrollando y matizando entre la comunidad cient ífica (Conelli y Clandinin, 1995). Y es que la narratología, ya sea como enfoque o como método de investigación e interpretación, puede contribuir a mejorar la comprensión del desarrollo personal. El enfoque narrativo posee, además, grandes posibilidades en el ámbito de la investigación educativa, puesto que puede indagar en el pensamiento y las acciones de los sujetos que establecen relaciones colaborativas y comprometidas éticamente, como se apunta en la línea de Colby y Damon. 
De esta forma, y aunque sea insistir, se debe resaltar que la comprensión de los motivos e intenciones que argumentan los sujetos, cuando se indaga en su historia de vida, nos posibilita un mayor conocimiento de su desarrollo personal. Así, la narrativa (historias) y la reflexión personal conforman un elemento central de una educación integral.

Relacionado con el carácter moral, tiene cabida el concepto de inteligencia moral, con el que Coles (1998) resalta que ser buena persona es signo de ser inteligente moralmente. Esta idea deriva, en parte, de la teoría acerca de la inteligencia emocional (Goleman, 1997), es decir, una forma de realizar interacciones en un mundo que tiene en cuenta los sentimientos y que engloba aspectos como habilidades de control de impulsos, empatía, entusiasmo, motivación, altruismo o compasión. Por este lado, Samay (1986) considera que la emoción moral es la esfera que provoca el dinamismo en la vida moral.

La teoría psicoanalítica basaba gran parte de su psicología moral en emociones como la culpabilidad y la vergüenza. En la sensibilidad al dolor ajeno reside el núcleo de la teoría de Hoffman (1987) de la socialización moral, y sirve de base relevante incluso en modelos derivados del desarrollo cognitivo tales como los de Gibbs (1991) y Selman (1980).

Tradicionalmente existen dos grandes categorías de emociones morales. La primera engloba lo que podemos denominar las emociones de la autocrítica, que son sentimientos de aversión que apuntan a algún tipo de autocensura o autovaloración negativa, como respuesta a un pensamiento o acción indeseable. Nos estamos refiriendo aquí a emociones tales como la culpabilidad, la vergüenza y el remordimiento. La segunda clase de emociones es de índole prosocial, y representan algún tipo de reacción afectiva ante la aflicción de los demás. Aludimos con ello a emociones tales como la empatía y la simpatía.

Al retomar la ya mencionada característica de la compasión, Ortega Ruiz y Mínguez Valleros (1999) proponen un modelo educativo basado en este componente de la personalidad, partiendo de que la teoría kohlberiana muestra una insuficiencia en cuanto a la consideración de este aspecto. En este sentido, las experiencias socio-afectivas, la adquisición de destrezas sociales y el desarrollo de la conciencia moral permiten a la persona la adquisición del rol de la compasión. La importancia de saber ponerse en el lugar del otro y simpatizar con diferentes personas es resultado de una sensibilidad moral y no, únicamente, de un ejercicio intelectual. En opinión de estos dos autores la educación en la compasión es la educación para la vida.

Asimismo la formación en la sensibilidad del alumno hacia valores como la tolerancia, la solidaridad, el respeto, de ir más allá, no sólo se consigue orientando a los educandos hacia aquellos, sino también con el objetivo de que sean capaces de sentirse satisfechos al comportarse según esos valores. Ya que ello, propicia una motivación hacia la conducta moral de forma generalizada.

Todas estas variables también se relacionan con el objetivo educativo crucial de adquirir un compromiso moral, que es uno de los factores que Hart y Killen (1995) resaltan en la explicación del juicio y acción moral. Así, por ejemplo, Hart, Yates, Fegley y Wilson (1995) examinan de qué manera los 
adolescentes deben de poseer una actitud de responsabilidad con su comunidad a través de compromisos sociales, como por ejemplo, con situaciones de empobrecimiento.

Enfoques tales como el de la Comunidad Justa de Kolhberg y otros modelos colectivistas, se centran asimismo en las sanciones del grupo de iguales, incluyendo la desaprobación, la expulsión y otras formas de castigo, así como modos de subrayar y reforzar los rasgos de carácter deseados, tales como la atención hacia los demás miembros de la comunidad y la participación en actividades de dicha comunidad, por ello se promueve aprender a vivir juntos, aprender a cooperar, aprender para la democracia y aprender para la diversidad.

Colby y Damon (1995) también subrayan la importancia del compromiso moral, desde el marco del ciclo vital, en el que podemos analizar cómo el compromiso va variando a lo largo de la vida, desde jóvenes a mayores, bajo la influencia de las situaciones.

Puig Rovira $(1995,1996)$ efectúa este planteamiento desde un análisis contextual y cultural, en donde la realidad social (revolución tecnológica y de la información, la crisis del capitalismo y del estatismo y los movimientos sociales) influyen en las dimensiones, estrategias y ámbitos temáticos de la educación moral. También considera un marco macrosocial para entender los valores, ya que, aunque se deben trabajar y defender unos valores mínimos básicos universales (basados en una ética de derechos humanos) y oponerse a los contravalores (en contra de esa ética básica), existen unos valores pers onal y socialmente controvertidos (por ejemplo, la eutanasia) que, en cierta manera, están muy condicionados por el contexto específico. Así, la amplitud de estos valores puede ir desde un ámbito de controversia personal hasta uno social. Este autor denomina micro-ético a problemas intraindividuales, meso-éticos a los que surgen en la convivencia de entornos cercanos al grupo (familia, amigos, escuela...) y macro-éticos a los relacionados con problemas sociales que nos atañen a todos. Esta división de Puig Rovira se asemeja a la que se plantea Cortés (2002), que enlaza el campo moral y el ecológico, en el que se conceptualizan y se analizan todas las temáticas morales en los cuatro niveles ecológicos (microsistema, mesosistema, exosistema, macrosistema) de Bronfenbrenner, concluyendo que todos los valores están condicionados e influenciados por los entornos más próximos incluso los más alejados como el macrosistémico. Aunque son los primeros, es decir, los contextos de desarrollo cercanos o microsistemas como la familia, sobre todo, el trabajo, la escuela, en los que más dilemas o conflictos sociéticos se suscitan frente a otras problemáticas de preocupación social más relacionadas con la cultura o macrosistema. Una propuesta de este resultado es la necesidad de trabajar de forma más notable y significativa en las comunidades, escuelas y familia para que las personas adquieran un compromiso social.

Esta responsabilidad es una característica de la sabiduría, que se aborda a través de la teoría del ciclo vital o life-span. Ésta subraya cómo los fenómenos psicológicos desde la infancia a la vejez están determinados por factores ontogénicos, cognitivos y sociales (Baltes, 1987) y que la persona está en continuo desarrollo hacia esa sabiduría, que podría identificarse con el de desarrollo moral integral puesto que refleja la permanente dialéctica de la persona para ir haciéndose más equilibrada o madura. Otras características de la sabiduría son (Baltes, 1987; Corral, 1997): búsqueda da la plenitud, consciencia de los propios conocimientos, adecuado uso de los mismos en el contexto, estilo dialéctico y dinámico, saber 
organizarse la vida, habilidad para integrar lo cognitivo, lo afectivo y la voluntad y conocimiento de los límites.

El esquema integral del desarrollo moral pretende relacionar las características de la psicología sociocultural y la cognitiva, y analizar cómo ambos planteamientos nos conducen al debate entre lo individual y contextual, al estudio de las estructuras y los procesos funcionales y de los esquemas sociales y sus aplicaciones en la vida. $Y$ todo ello aboga por el hecho de que la integración entre lo estructural y la perspectiva social en el desarrollo social cognitivo es posible. En este sentido, Del Río y Álvarez (1994) han trabajado los aspectos del desarrollo evolutivo desde una alternativa sociocultural y, desde ella, plantean estos tres postulados:

- La naturaleza histórica y socialmente cambiante de los sistemas de actividad en todas las etapas vitales (desde la infancia hasta la madurez y la senectud).

- Lo social como marco para entender los sistemas funcionales psicológicos.

- La necesidad de poner de relieve todas las narrativas culturales para el desarrollo sociomoral, la construcción de la identidad individual y social, y el desarrollo y educación general.

En posteriores estudios, estos dos autores, Del Río y Alvarez (1997) construyen una exposición en relación a tres ejes fundamentales: la identidad cultural, la personalidad moral y el saber. Se afirma que en el abordaje del pensamiento socio-moral se está pasando de una postura racional a un enfoque histórico y cultural. Es muy interesante el planteamiento que exponen acerca de los reduccionismos típicos de la psicología y cómo a éstos se les puede dar una respuesta desde el enfoque socio-cultural, como queda reflejado en este cuadro:

Cuadro 1: Integración desde el enfoque socio-cultural

\begin{tabular}{|l|l|}
\hline & Superación desde el enfoque sociocultural \\
\hline Racionalismo & La construcción cultural de la emoción, sentido y directividad. \\
\hline Individualismo & $\begin{array}{l}\text { La génesis de lo social de las funciones superiores y de la conciencia. Todo } \\
\text { lo que es individual antes ha sido social. }\end{array}$ \\
\hline Internista & $\begin{array}{l}\text { La relación sistémica y neural con el entorno cultural. Todo lo que es interno } \\
\text { antes ha sido externo. }\end{array}$ \\
\hline Innatista & Construcción social-histórico-cultural de las funciones superiores. \\
\hline
\end{tabular}

Con todas estas ideas, se quiere resaltar que como complemento al enfoque cognitivo-evolutivo, que enfatiza los componentes intelectuales, también es preciso incluir otros de tipo personal y contextual al extraer dilemas reales vitales, para ahondar más en la construcción de un modelo de integridad, referida a la consistencia entre juicio y acción moral, que caracteriza a la persona con una madurez moral (Walker, 1999). En otras palabras, Borrego de Dios (1999) propone tres ejes articuladores de dicho modelo: 1) promover la reflexión personal; 2) superar las lagunas entre el razonamiento y la actuación de tipo moral; 3) integrar las dimensiones convencionales y personales. Para ello se plantearía una globalización de lo propiamente moral, lo convencional y lo personal. 
En conclusión, se entiende la comprensión de la totalidad de la persona, desde un marco sistémico, con la relación y la conexión de aspectos como son el razonamiento, el carácter, el compromiso, la sensibilidad o inteligencia emocional, la compasión y la sabiduría vital. En el siguiente punto, se trata de entender qué intervención existe para educar en dichos aspectos, y se detalla en una propuesta denominada de Educación Holística.

\section{UNA RESPUESTA PARA EDUCAR EN EL DESARROLLO MORAL INTEGRO}

Esta pretensión se concreta en una educación global o total que es considerada como el nuevo paradigma educativo para el siglo XXI, y que se ha desarrollado a partir de la presente década de los noventa, recuperando el mejor conocimiento de diferentes campos e integrándolo con los nuevos desarrollos de la ciencia de la totalidad. Ofrece un genuino marco para entender el sentido de la educación en la nueva época, por un lado recupera lo mejor de los educadores clásicos y por el otro supera los falsos supuestos en que se basó la educación durante el siglo XX. El resultado es un paradigma educativo, enormemente creativo, sin precedentes en la historia de la enseñanza que está revolucionando radicalmente nuestras ideas sobre el proceso entre el docente y el discente.

Esta propuesta señala cuatro tipos de aprendizaje que es necesario desarrollar en las comunidades educativas del siglo XXI, y que son los siguientes: Aprender a aprender; Aprender a hacer; Aprender a vivir juntos y Aprender a ser. Estos cuatro aprendizajes se reconocen como estratégicos para los seres humanos del siglo XXI, y se orientan a las cuatro dimensiones de la educación holista: ciencia, sociedad, ecología y espiritualidad. El cambio de paradigma educativo puede comprenderse mejor siguiendo un análisis comparativo de las controversias entre una educación de tipo más tradicional-conductista-mecanicista-cognitivista, que se presentan en el siguiente cuadro:

Cuadro 2: Educación tradicional frente a la holística

\begin{tabular}{|l|l|}
\hline Reduccionista & Integral \\
\hline Metáfora guía: la máquina & Metáfora guía: organismos en red \\
\hline Multidisciplinariedad & Interdisciplinariedad \\
\hline División del conocimiento & Integración del conocimiento \\
\hline Conocimiento empírico - analítico & Conocimiento empírico - analítico - holístico \\
\hline Desarrollo del pensamiento & $\begin{array}{l}\text { Desarrollo de toda la personalidad, inteligencia, } \\
\text { lectividad. }\end{array}$ \\
\hline Cientificismo y/o dogmática & Espiritual \\
\hline Importancia de la enseñanza & Importancia del proceso de enseñanza-aprendizaje \\
\hline Currículum estático predeterminado & Currículum dinámico contextualizado \\
\hline Currículum centrado en disciplinas & Currículum centrado en preguntas relevantes \\
\hline Centrado sólo en la ciencia & Centrado en el conocimiento humano ciencia - arte - \\
\hline Indaga la dimensión externa y cuantitativa & espiritualidad - tradiciones \\
\hline Fundado en organizaciones burocráticas de & Indaga las dimensiones externa y cuantitativa, e \\
\hline enseñanza & Fundado en comunidades de aprendizaje \\
\hline Cambios superficiales de la conducta & Cambios profundos de la conciencia \\
\hline Paradigma de la simplificación & Paradigma de la complejidad \\
\hline
\end{tabular}


Yus (2001) ha realizado un trabajo sobre la educación integral partiendo de los diez principios que se promulgaron en el VIII Congreso Internacional de Educadores Holísticos, celebrado en 1990, y en el que crearon la Alianza Global para la Educación Transformadora (GATE).

El primer principio versa sobre una primacía del equilibrio entre lo humano sobre lo económico en el sentido de que sólo la persona plena e íntegra puede ser productiva en la sociedad. El respeto a la individualidad de cada sujeto es el segundo referente importante de esta declaración. Además, un tercer punto realza que el educando debe estar en contacto real con la vida laboral y social porque la experiencia es básica en el desarrollo, más aún cuando este se pretende que se contemple de forma holística, como se propone en el cuarto principio. Un quinto apartado se dirige a los profesores que deben potenciar una comprensión significativa de la complejidad del mundo, que exige que la educación sea más favorecedora de la libertad aportando alternativas para responder a una cultura heterogénea. Los principios VII, VIII y IX promueven, respectivamente, en primer lugar una educación para la democracia participativa en la que los ciudadanos puedan expresar en la comunidad y sepan ser críticos con ésta; en segundo lugar, la ciudadanía global para estar por encima de valores relativos y promulgar otros de índole más universal como el amor, compasión, sabiduría, verdad y armonía; y la alfabetización de la tierra que incluye procesos de cambio políticos, culturales y personales para entender desde una perspectiva global y ecológica la profunda interconexión de todas las microesferas de la vida. Y por último o desde el principio décimo, partir de la espiritualidad del sujeto o de su alma para poder trascender a otra dimensión más social, es decir, sólo partiendo de un desarrollo integro se podrá conseguir que la persona se preocupe por el resto de los individuos.

Los criterios pedagógicos que subyacen en esta educación holística, siguiendo a GATE y a Miller (1996), promueven una implicación y compromiso de la escuela en varios sentidos. En primer lugar, el estudiante es el centro del contexto de enseñanza-aprendizaje y como persona intrínsecamente buena, según dice Rousseau, debe de alcanzar una plenitud personal. Con este fin, se aboga por un currículum como medio para fomentar la transferencia en todas las áreas disciplinares y la escuela como sistema viviente y responsable de una comunidad más amplia.

El docente es uno de los responsables directos de la implantación de una educación basada en una "ideología" holista o global. Se habla de poseer y trabajar en cualidades psicológicas y sociales necesarias e imprescindibles para educar a los sujetos, que comienzan con una autenticidad psicológica (reales en la comunicación con los estudiantes), moral (coherentes entre lo que dicen y sus actos, sobre todo en el desarrollo de valores) y holística (creyentes de una educación holística para enseñar desde esta perspectiva). Se requiere que el maestro sea atento con las necesidades del educando desde una actitud de aceptación y confianza. Otra característica se refiere al cuidado con el alumno. En general, se pide que el profesor no sólo posea conocimientos, sino que sea una persona comprometida con su trabajo que es el de educar y formar personas íntegras y, para ello él debe ser también modelo y enseñar desde su Yo. El docente es un agente moral que debe: incitar la motivación de los alumnos en la participación de los procesos de la educación moral, crear un aula de tolerancia entre el alumnado, promover situaciones de diálogo y debate moral, procurar actitudes de comunicación y de escucha, saber ser mediador en los 
conflictos, seguir el proceso de crecimiento de la persona con respeto y paciencia, y aceptar con responsabilidad y humildad el papel de experto moral.

Siguiendo varias referencias teóricas (Lickona, 1991; Miller, 1996; Yus, 2001), el desarrollo holístico de la persona contiene facetas educativas interdependientes, que se engloban en tres áreas: personal, interpersonal y social. La primera recoge la educación para el cuerpo, las emociones, la creatividad, la individualidad, la experiencia, el carácter y el espíritu. La de índole interpersonal trabaja en la enseñanza hacia la justicia social y la democracia. Y la última propone una educación para el compromiso con la complejidad de la sociedad.

Un posible ejemplo de propuesta holística, se encuentra en la Universidad de Barcelona (Buxarrais, 1996; 2000), en la que se trabaja desde una perspectiva integradora un modelo de educación moral con el fin de formar personas coherentes entre el juicio y la acción moral, teniendo en cuenta tres componentes: cognitivo, emocional y volitivo, a fin de que estas sean felices. Para ello, este grupo de investigadores denominado GREM trabajan ocho aspectos de la personalidad moral: autoconocimiento, autonomía y autorregulación, capacidades para el diálogo, capacidad para transformar el entorno, la comprensión crítica, capacidad de empatía, las habilidades sociales y el razonamiento moral.

De esta manera, la educación holística no es un currículo o una metodología determinados; es un conjunto de proposiciones que incluye lo siguiente:

?? La educación es una relación humana dinámica y abierta.

?? La educación cultiva una conciencia crítica de los muchos contextos en la vida de los educandos: moral, cultural, ecológico, económico, tecnológico y político.

?? Todas las personas poseen vastos potenciales múltiples que solamente ahora estamos empezando a comprender. La inteligencia humana se expresa por medio de diversos estilos y capacidades, todos los cuales debemos respetar.

?? El pensamiento holístico incluye modos de conocer intuitivos, creativos, físicos y en contexto.

?? El aprendizaje es un proceso que dura toda la vida. Todas las situaciones de la vida pueden facilitar el aprender.

?? El aprendizaje es tanto un proceso interno de descubrimiento propio así como una actividad cooperativa.

?? El aprendizaje es activo, con motivación propia, que presta apoyo y estímulo al espíritu humano.

?? Un currículo holístico es interdisciplinar e integra las perspectivas globales y de la comunidad.

Por lo tanto, el desarrollo moral como desarrollo íntegro implica una concepción psicoeducativa del entendimiento del hombre como moral en sí mismo y unas referencias a nivel teórico y de intervención desde un aprendizaje y enseñanza holísticos. Esta idea se concreta con precisión en estas palabras:

“La educación holística reconoce que los seres humanos buscan significación, no solamente datos o destrezas, como aspecto intrínseco de un desarrollo completo y sano. Creemos que sólo seres humanos 
sanos y realizados pueden crear una sociedad sana. La educación holística cultiva las aspiraciones más altas del espíritu humano". (VII Congreso Internacional de Educadores Holísticos, 1990)

En resumen, en este trabajo se ha pretendido explicar la necesidad de educar a los sujetos bajo una perspectiva ética. Con este fin, se entiende que el desarrollo moral en su totalidad pasa por la confluencia de todos los componentes de la personalidad y del ambiente que rodea a los individuos. Es quizá éste el punto más significativo de toda la exposición, porque no nos atenemos a una característica únicamente, sino a varias, y además intentando conexionarlas de forma coherente ya que el individuo es una amalgama de razón, sentimiento y producto social. Y, por lo tanto, también esta situación se refleja en su faceta moral.

Se intenta estar lejos de una visión parcial, y así se comprende que para que una persona que esté madura éticamente necesita razonar hacia un equilibrio o razonamiento postconvencional, saber empatizar con otros sujetos, participar socialmente estando comprometidos y poseer un sentimiento de compasión. Todo ello supone un camino y un proceso en el que la educación es esencial porque la sociedad en la que habitamos y convivimos, con sus actuales circunstancias y problemáticas (inmigración, globalización económica, competitividad laboral y social, individualismo, corrupción...) requiere unos ciudadanos con una formación en esta línea ética.

Por último, para que cuaje este objetivo es necesario un modelo de enseñanza-aprendizaje con un currículum acorde y unas condiciones escolares adecuadas. Posiblemente, son necesarias más experiencias reales en centros educativos para saber cómo funciona este compendio teórico-práctico.. $Y$ quizá, sería necesario hacer hincapié, aunque ello suponga una dificultad, la pretensión de que estos "logros" de aprendizaje se generalicen a otros contextos, además del escolar, y se mantengan a largo plazo en el desarrollo de toda la vida de los que son niños en su escuela hasta cuando sean personas mayores.

\section{BIBLIOGRAFÍA}

Aierbe, A., Cortés, A. y Medrano, C. (2001). Una visión integradora de la teoría kohlberiana a partir de las críticas contextuales: propuesta de investigación. Cultura y Educación, 13 (2), 147-177

Aierbe, A., Cortés, A. y Medrano, C. (2000). La experiencia moral en los adolescentes: historias de vida y dilemas reales. En E, Marchena Consejeros y C, Alcala Cuevas (comp.), La perspectiva de la educación en el siglo que empieza. (pp 341-358) Servicio de Publicaciones de la Universidad de Cadiz: Cadiz.

Baltes, PB. (1987). Theorical propositions of life-span developmental psychology: on the dynamics between growth and decline. Developmental Psychology, 23, 5, 611-626.

Bennett, W.J. (1991). Moral literacy and the formation of character. In J.S. Benninga (comp.), Moral, character, and civic education in the elementary school (pp. 131-138). New York: Teachers College Press.

Bennett, W.J. (1993). Book of virtues. Bellevue, WA: S \& S Trade.

Borrego de Dios, S. (1999): Desarrollo socio-personal y moral: la cara interna de la educación global. Investigación en la escuela, 37, 85-98.

Buxarrais, MR. (1996). La formación del profesorado en educación de valores. Bilbao: Desclée De Brouwer. 
Buxarrais, MR. (2000). Perspectivas actuales en la educación moral. En C, Medrano y I, Elexpuru, Actas del Curso de Verano 2000: Los valores desde la perspectiva educativa.

Colby, A. y Damon, W. (1994). Some do Care. New York: The Free Press.

Colby, A. y Damon, W. (1995). The development of extraordinary moral comminent. In Killen, M y Hart, D (comp.), Morality in everyday life (pp 342-370). Cambrigde: Cambrigde University Press.

Coles, R. (1998). The moral intelligence of children. London: Bloomsbury.

Conelli, MF. y Clandinin, DJ. (1995). Relatos de experiencia e investigación narrativa. En J, Larrosa y otros (comp.), Dejame que te cuente. Ensayos sobre narrativa y educación. Barcelona: Laertes.

Corral, A. (1997). Las operaciones formales y postformales en la vida adulta. En JA, García Madruga y P.León (comp.), Psicología Evolutiva II (pp 317-348). Madrid: UNED.

Cortés Pascual, A (2002). Hacia un modelo global de desarrollo moral desde Kohlberg y Bronfenbrenner. Bilbao: Servicios de publicaciones de la UPV.

Del Río, P. y Alvarez, A. (1994). Introducción: La educación como construcción cultural en un mundo cambiante. En A, Álvarez; P, Del Rio y J, Wertsch, (comp.). Explorations in Socio-Cultural Studies (pp 239-253) (vol.4). Madrid: Fundación Infancia y Aprendizaje.

Del Rio, P. y Alvarez, A. (1997): ¿Saber o comportarse?. El desarrollo y la construcción de la directividad. En A, Alvarez (comp.), Hacia un curriculum cultural: la vigencia de Vygotsky en la educación (pp 101 131). Madrid: Fundación Infancia y Aprendizaje.

Gibbs, J.C. (1991). Toward an integration of Kohlberg's and Hoffman's theories of morality. In W.M. Kurtines, \& J.L. Gewirtz (comp.), Handbook of moral behavior and development. Volume 1: Theory (pp. 183222).

Goleman, D. (1997): Inteligencia Emocional. Barcelona: Kairós.

Hart, D. y Killen, M. (1995). Introduction: perspectives on morality in everyday life. In Killen, M y Hart, D (comp.), Morality in everyday life (pp 1-19). Cambrigde: Cambrigde University Press.

Hart, D., Yates, M., Fegley, S. y Wilson, G. (1995). Moral commitment in inner-city adolescents. In Killen, M y Hart, D (comp.), Morality in everyday life (pp 317-341). Cambrigde: Cambrigde University Press.

Hoffman, M.L. (1987). The contribution of empathy to justice and moral judgment». In N. Eisenberg \& J. Strayer (comp.), Empathy and its development. New York: Cambridge University Press.

Kohlberg, L. (1992). La psicología del desarrollo moral (volumen 2). Bilbao: Desclée de Brouwer. (Orig.1984)

Lickona, T. (1991). An integrated approach to character development. In J.S. Benninga (comp.), Moral, character, and civic education in the elementary school (pp. 67-83). New York: Teachers College Press.

Lickona, T. (1992). Educating for carácter: how our schools can teack respect and responsability. New York: Bantan Books.

Lickona. T (1996). Eleven principles of effective character education. Journal of moral education, 25, 93-100.

Miller, (1996). The Holistic Teacher. Toronto: OISE Press.

Muñoz Redón, J. (1999). Filosofía de la Felicidad. Barcelona: Anagrama.

Ortega Ruiz, P. y Minguez Vallejos, R. (1999). The role of compassions in moral educations. _, 28, 1, 5-17

Papalia, DE. y Wandkos Olds, S. (1997). Desarrollo humano. Santafé de Bogota: Mc Graw Hill. 
Puig Rovira, JM. (1995). La educación moral en la enseñanza obligatoria. Cuadernos de educación, 17. Barcelona: Horsori.

Puig Rovira, JM. (1996). La construcción de la personalidad moral. Barcelona: Paidos.

Samay, S.A. (1986). Affectivity: The power base of moral behavior. In G.F. McLean, F.E. Ellrod, D.L. Schindler, \& J.A. Mann (comp.), Act and agent: Philosophical foundations for moral education and character development (pp. 71-114). New York: University Press of America.

Selman, R. (1980). The growth of interpersonal understanding. New York: Academic Press.

Yus, R (2001). Educación Integral. Una educación holística para el siglo XXI. Volumen I y II. Bilbao: Desclée de Brouwer.

Walker, L. J. (1999). The percived personality of moral exemplars. Journal of Moral Education, 28, 3, 261 264. 